\title{
BENEFÍCIOS DO TRATAMENTO DE PESSOAS COM A SÍNDROME DA APNEIA OBSTRUTIVA DO SONO
}

\section{ARTIGO DE REVISÃO}

SOUZA, Dalton Berbek de ${ }^{1}$

CARVALHO, Alessandra Alonso de ${ }^{2}$

OLIVEIRA, Amanda Simoso do Nascimento de ${ }^{3}$

ROCHA, Marcela Pioto ${ }^{4}$

SOUZA, Dalton Berbek de. CARVALHO, Alessandra Alonso de. OLIVEIRA, Amanda Simoso do Nascimento de. ROCHA, Marcela Pioto. Benefícios do tratamento de pessoas com a Síndrome da Apneia Obstrutiva do Sono. Revista Científica Multidisciplinar Núcleo do Conhecimento. Ano 05, Ed. 01, Vol. 10, pp. 102-118. Janeiro de 2020. ISSN: 2448-0959, Link de acesso: https://www.nucleodoconhecimento.com.br/saude/beneficios-do-tratamento

\section{RESUMO}

A síndrome da apneia obstrutiva do sono é um distúrbio relacionado a despertares durante o sono recorrentes, e, em muitas das vezes, mal diagnosticado. O objetivo da pesquisa é descrever os benefícios que o CPAP pode trazer como forma de tratamento aos portadores da SAOS. Para isso, conhecer o perfil dos pacientes e verificar as possíveis complicações é recomendado. O método científico utilizado foi o de revisão bibliográfica de caráter exploratório. Foram usados como fonte de informações as publicações cientificas das bases de dados da Biblioteca Virtual de

${ }^{1}$ Enfermeiro Graduado pela Universidade Anhembi Morumbi - SP.

2 Enfermeira pela Universidade Anhembi Morumbi- SP.

${ }^{3}$ Enfermeira pela Universidade Anhembi Morumbi- SP.

${ }^{4}$ Enfermeira, Docente de Enfermagem da Universidade Anhembi Morumbi- SP. 
Saúde - BVS, Scientific Eletronic Libery Online - SCIELO e da Literatura Latino Americana e do Caribe em Ciências da Saúde - LILACS dos anos de 2013 a 2017. Os estudos destacam que o CPAP é a primeira opção de tratamento para os portadores da SAOS. Se o paciente aderir o tratamento de forma correta, ocorrerá melhora do controle glicêmico, diminuição nos episódios de roncos, melhora da qualidade do sono e a prevenção de possíveis riscos cardiovasculares. Em vista dos argumentos apresentados, gostaríamos de destacar a relevância do assunto abordado nesta pesquisa, pois é um tema pouco explorado e de alta prevalência na sociedade. Percebemos que, provavelmente, os números levantados em pesquisas são errôneos e menores do que a realidade.

Palavras-chave: Síndrome da apneia obstrutiva do sono, terapêutica, qualidade de vida.

\section{INTRODUÇÃO}

A Síndrome da Apneia Obstrutiva do Sono (SAOS) é considerada uma doença crônica, recorrente, e, em muitas das vezes, é mal diagnosticada. Está relacionada a esforços inspiratórios ineficazes, o que ocasiona na oclusão repetitiva das vias aéreas superiores (VAS) e pausas respiratórias, durando, em média, dez segundos ou até mais. São capazes, ainda, de fazer o indivíduo acordar por sufocamento durante o sono (LEITE et al, 2017; LACERDA et al, 2013; ANDRADE; PEDROSA, 2016). Esse grave distúrbio obstrutivo das vias aéreas fragmenta a qualidade do sono e, dessa forma, afeta a qualidade de vida do portador, elevando os índices de mortalidade, além de predispor o indivíduo às doenças de base, às alterações cardiovasculares e metabólicas e ao comprometimento do sistema nervoso central (SNC) (NASCIMENTO et al, 2014; ANDRADE; PEDROSA, 2016).

O sono é considerado como um estado fisiológico com integração ao sistema nervoso central e compõe o ciclo sono-vigília seguindo uma frequência circadiana, tendo uma variabilidade na idade, sexo e outras características fisiológicas. Há, ainda, uma normatização do ciclo por meio dos processos homeostático e circadiano (ALVES; RAMOS; VOLPATO, 2016; NEVES; MACEDO; GOMES, 2017). Em busca de novos 
trabalhos que procuram descrever e avaliar os benefícios dos pacientes que sofrem com a síndrome da apneia do sono, buscou-se verificar quais são os benefícios que o CPAP traz para os portadores da SAOS em uso do dispositivo. Detectou-se que o CPAP beneficia o paciente que sofre com apneia do sono, pois há a diminuição das interrupções da respiração, aumento da saturação da oxiemoglobina, redução dos despertares relacionados aos eventos respiratórios e diminuição da sonolência durante o dia.

A SAOS é considerada um grave problema de saúde, devido à, principalmente, os altos índices de mortalidade, aumentando, então, riscos de acidentes, perda de memória, cansaço, além de estar relacionada com problemas cardiovasculares (LACERDA et al, 2013; ANDRADE; PEDROSA, 2016). O presente trabalho justificase por ser um tema com grandes vertentes para pesquisa. Estudos referem que uma parcela considerável de 20 a 80 anos da população mundial adulta é afetada por transtornos relacionados ao sono. Estima-se que $32,8 \%$ da população geral sofre com distúrbios relacionados ao sono, além disso existe uma alta porcentagem de 40,6\% dos homens serem acometidos pela doença (ANDRADE; PEDROSA, 2016). O objetivo deste estudo é descrever os benefícios que o CPAP pode trazer como forma de tratamento aos portadores da SAOS, conhecendo, para isso, o perfil dos pacientes para verificar as possíveis complicações.

\section{A SÍNDROME DA APNEIA OBSTRUTIVA DO SONO}

A Síndrome da Apneia Obstrutiva do Sono (SAOS) é um distúrbio ocasionado por recorrentes episódios de obstrução total das vias aéreas que fragmentam o sono, durando, em média, 10 segundos. Há, no mínimo, 5 episódios por hora de sono. Dessa forma, afeta a qualidade de vida do portador, pois os efeitos também são percebidos durante o dia e há uma alta predisposição às doenças de base, cardiovasculares, cognitivas e de memórias, além de outros agravos na qualidade de vida (ALVES; RAMOS; VOLPATO, 2016; TASSINARI et al, 2016). Estudos referem que a SAOS é uma patologia com altos índices de prevalência na população adulta (32,9\% da população entre homens e mulheres), sendo este um índice que pode favorecer o surgimento de outras patologias (ALVES; RAMOS; VOLPATO, 2016; 
TASSINARI et al, 2016). O sono é fundamental pois tem função regeneradora, protetora, imunológica e conserva energia. O ciclo de sono-vigília segue a frequência do ciclo circadiano e depende de características individuais.

Ele é gerado a partir de dois processos que estão intimamente relacionados: o processo homeostático, no qual são liberadas substâncias que geram o sono após grande período de vigília e o processo circadiano que tem a ver com a variação cíclica da vigília e do sono durante o dia, regulado pelo marca-passo circadiano, que é o núcleo supraquiasmático, sendo dependente de funções endógenas e de variações ambientais, como a luminosidade (NEVES; MACEDO; GOMES, 2017). Ao despertarmos, entra em ação o processo circadiano, que gera influências excitatórias no córtex cerebral. A retina capta estímulos luminosos que atingem o núcleo supraquiasmático e controlam a secreção de melatonina. Esta volta a ser secretada entre 1 a 3 horas antes do sono e tem seu pico durante o ponto mais baixo da temperatura central corporal (NEVES; MACEDO; GOMES, 2017).

O sono apresenta duas fases fundamentais: sem movimentos oculares (NREM) com 4 estágios (75\%) e com movimentos oculares (REM) com 1 estágio (25\%). O sono NREM é caracterizado por ondas lentas e pela redução da atividade muscular e o sono REM é caracterizado por movimentos oculares involuntários, pela presença de sonhos e pela consolidação de informações aprendidas durante o dia (NEVES et al, 2013). O sono se inicia pela fase NREM e após aproximadamente 90 minutos é iniciada a fase REM, formando, assim, o ciclo NREM-REM. Nele ocorre, aproximadamente, de 5 a 6 ciclos em um período de 8 horas (NEVES et al, 2013). O diagnóstico precoce da SAOS fornece diretrizes para um tratamento eficaz, de acordo com à gravidade da doença, idade e condições sistêmicas do paciente.

Os pacientes acometidos pela doença apresentam aspectos clínicos que devem ser investigados. São eles: fadiga, hipersonolência diurna, irritabilidade, diminuição da libido, impotência e cefaleia matinal intensa, além disso a síndrome é frequentemente associada a acidentes automobilísticos. Na maioria das vezes, a primeira consulta é motivada por reclamações do cônjuge a respeito dos roncos altos e frequentes (SILVA et al, 2014; PACHECO; ANJOS; MAIA, 2015). Alguns instrumentos de aferição foram 
criados com o intuito de serem usados na prática clínica como alternativa à polissonografia. Auxiliam, dessa forma, no diagnóstico clínico, visto que possuem um menor custo. O principal deles é o Questionário de Berlim (BQ). Ele foi criado para rastrear pacientes com risco de SAOS. É um questionário de fácil entendimento que avalia a ocorrência de sintomas como: ronco, sonolência diurna, obesidade, fadiga e hipertensão arterial (MOURA et al, 2017).

Outra forma de diagnóstico é por meio do exame físico. A partir dele avalia-se o peso, altura, circunferência do pescoço e a pressão arterial. A polissonografia (PSG) é o método padrão ouro para o diagnóstico da SAOS. Mesmo sendo evidenciado clinicamente, seu diagnóstico só pode ser confirmado por meio do (PSG) (SILVA et al, 2014). Ele permite a identificação do número e a duração das respirações com alterações, além de favorecer a observação dos efeitos da respiração anormal sobre a saturação da hemoglobina e se há o aumento do ritmo cardíaco nas diferentes fases do sono. Com isso é possível classificar a gravidade da SAOS. O exame tem a finalidade de mensurar a quantidade e a qualidade do sono do paciente. Apesar deste ser o exame essencial para o diagnóstico, não é capaz de identificar o local da obstrução, por isso é necessário ser associado a exames de imagens. Assim, é possível visualizar o local de estreitamento das vias aéreas (SILVA et al, 2014; PACHECO; ANJOS; MAIA, 2015).

\subsection{TRATAMENTO: CPAP (CONTINUOUS POSITIVE AIRWAY PRESSURE)}

O tratamento com pressão positiva contínua na via aérea evita que ocorra o "fechamento" da via aérea superior, porque conta com um fluxo de ar contínuo e constante, e quando usado de forma contínua e correta, propicia uma qualidade de vida mais positiva para o paciente pois: melhora o controle glicêmico, há a estabilização metabólica (por melhora da oxigenação), diminui episódios de roncos, engasgo, sono fragmentado, danos cardiovasculares, dentre outros benefícios (JÚNIOR; FILHO; DRAGER, 2013). A aderência ao tratamento é definida pelo uso de, no mínimo, 4 horas por noite, sendo esse dado apenas uma convenção, pois não foi 
definido o tempo necessário para controle dos sintomas da SAOS. Deve ser feita uma indicação adequada sobre a modalidade ventilatória, assim, a máscara apropriada deve agradar o paciente para que consiga se adaptar e para que a pressão efetiva previna a ocorrência de apneias e ronco.

Se essa pressão for inadequada pode ocasionar em lesões cutâneas ou no ressecamento das mucosas. O paciente deve ser orientado sobre o uso da máscara e sobre a técnica de aplicação assim como quanto à higiene dos equipamentos (NOGUEIRA; BORSINI; NIGRO, 2016). É função primordial do profissional de saúde monitorar efeitos adversos, controlar a ansiedade, educar o paciente quanto à necessidade e importância do tratamento, orientar possíveis riscos que podem ocorrer com a falta dele, criar vínculo, sanar dúvidas e esclarecer o quadro clínico ${ }^{10}$. Vale ressaltar que o CPAP não cura a SAOS, mas seu uso gera muitas vantagens, como a normalização do padrão respiratório do sono ao eliminar os episódios de apneia. Há, também, melhora do excesso de sonolência diurna, estabilização e regressão de doenças cardiovasculares relacionadas com a SAOS (LACERDA et al, 2013).

A SAOS é um dos mais comuns distúrbios do sono, porém é de difícil diagnóstico e grande parte da população é portadora sem ter o conhecimento. Estudos estimam que "a prevalência da SAOS seja de aproximadamente 3\% a 7\% em homens e $2 \%$ a $5 \%$ em mulheres, contudo esta taxa pode ascender a valores mais elevados em grupos de risco". Essa síndrome pode afetar qualquer faixa etária, porém a probabilidade de ser acometido aumenta com o avanço da idade. Segundo a literatura, a SAOS é de 2 a 3 vezes mais frequente em homens do que em mulheres. Existem fatores que podem predispor a doença, são eles: obesidade, tabagismo, pressão arterial sistêmica, dentre outros (CAMPOSTRINI; PRADO; PRADO, 2014; PEREIRA, 2015).

\subsubsection{COMPLICAÇÕES E AGRAVOS}

A SAOS é uma doença crônica, e, se não tratada corretamente, pode acarretar consequências graves para os seus portadores, causando alterações comportamentais, cardiovasculares e neurológicas progressivas (SILVA et al, 2014). 


\subsubsection{HIPERTENSÃO ARTERIAL SISTÊMICA}

A SAOS e a hipertensão arterial são doenças comuns na população, sendo diagnosticadas ao mesmo tempo em um único paciente de forma significativa. Essa associação das duas patologias está relacionada ao fato de que a SAOS, frequentemente, ativa o sistema simpático dos indivíduos acometidos. Essa ativação gera uma diminuição dos barorreceptores, o que ocasiona na alteração do metabolismo da água e do sal e, consequentemente, ocorre a elevação da pressão arterial do paciente (CAMPOSTRINI; PRADO; PRADO, 2014). Vários estudos têm demonstrado a associação entre SAOS e HAS, estimando-se que cerca de $50 \%$ a $70 \%$ dos pacientes com SAOS sejam hipertensos e que $30 \%$ dos hipertensos tenham SAOS (CAMPOSTRINI; PRADO; PRADO, 2014).

\subsubsection{INSUFICIÊNCIA CARDÍACA}

A prevalência de SAOS entre pacientes portadores de insuficiência cardíaca varia de 10\% a 35\% (CAMPOSTRINI; PRADO; PRADO, 2014). A insuficiência cardíaca está altamente relacionada a HAS e essa alteração explica a disfunção sistólica do ventrículo esquerdo. Outro fator relacionado é a liberação de mensageiros químicos do Sistema Nervoso Central (SNC) juntamente com fatores envolvidos na fisiopatologia da SAOS. Eles contribuem para com o surgimento de uma hipertrofia do ventrículo esquerdo e isso independe do paciente ser hipertenso.

Os portadores de SAOS sempre sofrem hipóxia noturna, decorrente do fechamento ou estreitamento das vias aéreas e isso acaba acarretando a isquemia e apoptose nos miócitos, o que ocasiona, consequentemente, no relaxamento ventricular durante a diástole. Esse ciclo se repetindo ao longo da vida, e, dessa forma, gera um estresse das paredes e ocorre a dilatação dos ventrículos, trazendo complicações aos portadores a longo prazo (CAMPOSTRINI; PRADO; PRADO, 2014). 


\subsubsection{ARRITMIAS CARDÍACAS}

Segundo os especialistas da American Heart Association e da American College of Cardiology Foundation, as arritmias são registradas em até $50 \%$ dos doentes com SAOS e relacionam-se com o número de eventos de apneia e gravidade da diminuição de SaO2 associada (PEREIRA, 2015). No período de sono, há variações entre o sistema nervoso simpático e parassimpático dos portadores da SAOS, o que ocasiona arritmias. Quando o parassimpático é ativado, favorece-se as bradiarritmias, já quando o simpático é ativado há a fibrilação auricular e taquiarritmias ventriculares. Os fatores que levam as arritmias ventriculares incluem a hipóxia durante o sono, alterações do sistema nervoso simpático e hipertensão arterial sistêmica (PEREIRA, 2015).

\subsubsection{DOENÇA VASCULAR CEREBRAL}

A doença vascular cerebral é bem frequente na população e representa a segunda causa de morte no mundo. As alterações levam o paciente a momentos de hipóxia durante o sono devido ao colabamento ou estreitamento das vias aéreas. Com o passar dos anos isso gera danos às células e predispõe o desenvolvimento de doença vascular cerebral em portadores da síndrome. Quando o paciente tem a SAOS e desenvolve problemas vasculares o prognóstico tende a ser mais negativo e o risco de mortalidade aumenta, por isso é de extrema importância o rápido diagnóstico e implantação do tratamento adequado (PEREIRA, 2015).

\subsubsection{CONSEQUÊNCIAS PSICOSSOCIAIS}

A SAOS é uma doença complexa e que gera um grande impacto na vida do portador, pois diminui a sua qualidade de vida e ocasiona no seu afastamento da vida social, o que torna a doença relevante em termos de saúde mental e pública (PACHECO; ANJOS; MAIA, 2015). Após o diagnóstico da Síndrome da Apneia Obstrutiva do Sono, se o tratamento com o CPAP for iniciado, ocorre melhora dos sintomas, pois o excesso da sonolência diurna é minimizado, reduzindo, assim, os riscos de acidentes automobilísticos, há a diminuição das apneias e dos despertares noturnos, há melhora 
da capacidade funcional do indivíduo, o desempenho subjetivo no trabalho é positivo, a função cognitiva é mais notável e, consequentemente, há melhora da qualidade de vida (LACERDA et al, 2013).

Estudos realizados demonstraram que o tratamento em portadores da SAOS com o CPAP pode ocasionar queda da pressão arterial tanto em normotensos quanto nos hipertensos e se tratado em sua forma inicial previne o surgimento da Hipertensão Arterial Sistêmica (HAS). O uso do CPAP demonstrou redução de hospitalização e mortalidade em portadores de SAOS grave com insuficiência cardíaca. Após o Acidente Vascular Encefálico (AVE) a reabilitação foi melhorada com seu uso. Houve, também, melhora do metabolismo e redução do colesterol total, triglicerídeos e hemoglobina glicada, pois, em pacientes diabéticos, houve uma queda significativa. Detectou-se, ainda, que o tratamento a longo prazo (por volta de 7 anos) previne problemas cardiovasculares (JÚNIOR; FILHO; DRAGER, 2013).

O papel do exercício físico tem sido mais um aliado contra a SAOS, visto que acarreta em modificações metabólicas e estruturais e na elevação da temperatura, facilitando, assim, a chegada de mecanismos indutores do sono, além de que há uma maior duração do sono em estágio de ondas lentas, redução do peso e aumento do trabalho respiratório, principalmente do diafragma. Estudos expuseram que o maior estímulo do sistema respiratório decorrente da atividade física enrijece a musculatura, acarretando, diretamente, no aumento da resistência muscular, pois há, proporcionalmente, o aumento do diâmetro dessas vias, possibilitando, consequentemente, a resistência do fluxo de ar, o que impede o colapso da via aérea durante o sono (ANDRADE; PEDROSA, 2016).

Outras recomendações importantes para o aumento da qualidade de vida é a mudança de hábitos, como, por exemplo, a substituição ou erradicação de drogas que causam danos no funcionamento da musculatura das vias aéreas superiores, diminuição no consumo de bebidas alcoólicas, principalmente no período da tarde, a prática de atividades físicas e mudança do posicionamento do corpo durante o sono, evitando, sobretudo, a posição em decúbito dorsal (ANDRADE; PEDROSA, 2016). 


\section{METODOLOGIA}

O método científico utilizado foi o de revisão bibliográfica de caráter exploratório. Foram utilizadas como fonte as publicações cientificas das bases de dados da Biblioteca Virtual de Saúde (BVS), Scientific Eletronic Libery Online (SCIELO) e Literatura Latino Americana e do Caribe em Ciências da Saúde (LILACS) dos anos de 2013 a 2017 com os descritores: síndrome da apneia obstrutiva do sono, qualidade de vida e terapêutica. A coleta de dados para este estudo foi norteada a partir de cinco etapas. A primeira etapa foi a identificação do tema de pesquisa e seleção da pergunta de pesquisa; a segunda contemplou a discussão dos critérios de seleção e exclusão; a terceira foi a escolha dos artigos selecionados; a quarta a categorização dos artigos e a quinta etapa a análise, explanação dos resultados e apresentação da revisão. $\mathrm{Na}$ primeira etapa foi escolhida a pergunta norteadora do estudo para investigação do tema: Quais os benefícios que o CPAP traz para os portadores da SAOS ao usar o dispositivo?

$\mathrm{Na}$ segunda etapa os artigos utilizados para coleta de dados foram extraídos das bases de dados BVS, SCIELO e LILACS com os descritores: síndrome da apneia obstrutiva do sono, qualidade de vida e terapêutica. Na terceira etapa os critérios de seleção e exclusão pesquisados foram: 1- periódicos publicados nos anos de 2013 a 2017, 2- seleção de materiais que abordam a temática pesquisa e 3- artigos publicados na íntegra. Foram excluídos artigos que não atenderam os critérios de seleção acima. Quinze dele foram incluídos. Na quarta etapa, após a leitura e fixação de dos artigos selecionados, foram categorizados a partir do instrumento de coleta de dados validado por Bertolazi (2008). As categorias estabelecidas ressaltam os principais riscos da SAOS e os benefícios da aderência ao tratamento. Obtidos os resultados e evidências, os estudos foram sequenciados e deu-se início à construção da temática e enriquecimento do estudo. 
Figura 1: Etapas metodológicas da pesquisa

\section{$1^{\circ}$ Escolha da pergunta norteadora}

\section{$2^{\circ}$ Coleta de dados}

\section{$3^{\circ}$ Critérios de seleção e exclusão}

\section{$4^{\circ}$ Categorização dos estudos e início do estudo}

Fonte: Elaborado pelos autores (2019).

\section{RESULTADOS}

Na presente revisão da literatura foram analisados quinze artigos que atenderam aos critérios de inclusão concentrados nos períodos de 2013 a 2017. Detectou-se que a maioria dos artigos foram publicados em bases de dados e periódicos do Brasil, sendo a grande maioria dos pesquisadores profissionais da área da saúde. Ao verificar a abordagem, percebemos que esses autores utilizaram abordagens metodológicas quantitativas e qualitativas. Houve uma ênfase nos métodos descritivos.

Quadro 1: Identificação da amostra dos estudos segundo referências, autores, título e objetivos. São Paulo - SP, Brasil, 2019.

\begin{tabular}{|c|c|c|c|}
\hline Código e Ano & AUTORES & TÍTULO & OBJETIVOS \\
\hline \multirow{7}{*}{2017} & Adson R Leite, & Correlação de & Investigar há associação \\
\hline & & Risco entre & da presença do risco de \\
\hline & Erica A Macedo, & Síndrome $\quad \mathrm{da}$ & SAOS com alterações \\
\hline & Antonio JI Jorae & Apneia Obstrutiva & cardíacas identificadas \\
\hline & , & do Sono e & pelo \\
\hline & Maria L G Rosa, & Insuficiência & ecodopplercardiograma \\
\hline & & $\begin{array}{l}\text { Cardíaca } \quad \text { na } \\
\text { atenção }\end{array}$ & em pacientes sem \\
\hline
\end{tabular}




\begin{tabular}{|c|c|c|c|}
\hline & & & $\begin{array}{l}\text { sintomas de IC (estágios } \\
\text { A e B). } \\
\text { Ajudar na compreensão } \\
\text { da relação entre as duas } \\
\text { síndromes }\end{array}$ \\
\hline 2017 & $\begin{array}{ll}\text { Flávio } & \text { M } \\
\text { Andrades, } & \\
\text { Rodrigo } & \\
\text { Pedrosa } & \end{array}$ & $\begin{array}{l}\text { O papel do } \\
\text { exercício físico na } \\
\text { apneia obstrutiva } \\
\text { do sono }\end{array}$ & $\begin{array}{l}\text { Avaliar o papel do } \\
\text { exercício físico realizado } \\
\text { de forma isolada no } \\
\text { tratamento da SAOS. }\end{array}$ \\
\hline 2016 & $\begin{array}{l}\text { Paulo R R Alves } \\
\text { Fernando A } \\
\text { Ramos } \\
\text { Thaise B Volpato }\end{array}$ & $\begin{array}{l}\text { Qualidade de vida } \\
\text { em indivíduos com } \\
\text { apneia obstrutiva } \\
\text { do sono moderada } \\
\text { a grave antes e } \\
\text { após tratamento } \\
\text { com pressão } \\
\text { positiva continua } \\
\text { nas vias aéreas }\end{array}$ & $\begin{array}{l}\text { Analisar a qualidade de } \\
\text { vida dos indivíduos } \\
\text { portadores da SAOS } \\
\text { após o tratamento com o } \\
\text { CPAP. }\end{array}$ \\
\hline 2017 & $\begin{array}{l}\text { Walter L Moura } \\
\text { Carolina S Moura } \\
\text { Thalisson S O } \\
\text { Silva Graciela M O } \\
\text { Sipaúba } \\
\text { Mariá S L Moura } \\
\text { Gregório A S } \\
\text { Martins }\end{array}$ & $\begin{array}{lr}\text { Prevalência } & \text { do } \\
\text { risco da Síndrome } \\
\text { da } & \text { Apneia } \\
\text { Obstrutiva } & \text { do } \\
\text { Sono } & \text { na } \\
\text { população } & \text { adulta } \\
\text { de uma } & \text { capital } \\
\text { brasileira } & \end{array}$ & $\begin{array}{l}\text { Estimar a prevalência de } \\
\text { alto risco da Síndrome } \\
\text { da Apneia Obstrutiva do } \\
\text { Sono e fatores } \\
\text { associados, em adultos } \\
\text { de uma capital do } \\
\text { nordeste brasileiro. }\end{array}$ \\
\hline 2015 & $\begin{array}{lll}\text { Fernanda } & \text { Y } & R \\
\text { Pacheco } & & \end{array}$ & $\begin{array}{l}\text { Síndrome da } \\
\text { apnéia/hipopnéia } \\
\text { obstrutiva do }\end{array}$ & $\begin{array}{l}\text { Compreender a } \\
\text { síndrome da apnéia e } \\
\text { hiponéia obstrutiva do }\end{array}$ \\
\hline
\end{tabular}




\begin{tabular}{|c|c|c|c|}
\hline & $\begin{array}{l}\text { Elizabete S Anjos } \\
\text { André B F Maia }\end{array}$ & $\begin{array}{l}\text { sono: artigo de } \\
\text { revisão } \\
\text { bibliográfica }\end{array}$ & $\begin{array}{l}\text { sono em sua extensão, } \\
\text { complexividade } \\
\text { gravidade, englobando } \\
\text { desde } \\
\text { características } \\
\text { resoluções. }\end{array}$ \\
\hline 2014 & $\begin{array}{l}\text { Daniella D. A } \\
\text { Campostrini, } \\
\text { Lucila B. F Prado, } \\
\text { Gilmar F Prado }\end{array}$ & $\begin{array}{l}\text { Síndrome da } \\
\text { Apneia Obstrutiva } \\
\text { do Sono e } \\
\text { Doenças } \\
\text { Cardiovasculares }\end{array}$ & $\begin{array}{l}\text { Abordar aspectos } \\
\text { clínicos, fisiopatológicos } \\
\text { e diagnósticos da SAOS, } \\
\text { bem como seus efeitos } \\
\text { deletérios no sistema } \\
\text { cardiovascular. }\end{array}$ \\
\hline 2017 & $\begin{array}{l}\text { Walter L Moura } \\
\text { Carolina S Moura } \\
\text { Thalisson S O } \\
\text { Silva Graciela M O } \\
\text { Sipaúba } \\
\text { Mariá S L Moura } \\
\text { Gregório A S } \\
\text { Martins }\end{array}$ & $\begin{array}{lr}\text { Prevalência } & \text { do } \\
\text { risco da Síndrome } \\
\text { da } & \text { Apneia } \\
\text { Obstrutiva } & \text { do } \\
\text { Sono } & \text { na } \\
\text { população } & \text { adulta } \\
\text { de uma } & \text { capital } \\
\text { brasileira }\end{array}$ & $\begin{array}{l}\text { Estimar a prevalência de } \\
\text { alto risco da Síndrome } \\
\text { da Apneia Obstrutiva do } \\
\text { Sono e fatores } \\
\text { associados, em adultos } \\
\text { de uma capital do } \\
\text { nordeste brasileiro. }\end{array}$ \\
\hline \multirow[t]{2}{*}{2016} & $\begin{array}{l}\text { Nogueira Juan } \mathrm{F}^{1} \text {, } \\
\text { Borsini Eduardo }{ }^{2} \text {, } \\
\text { Nigro Carlos }{ }^{3}\end{array}$ & $\begin{array}{lr}\text { Estratégias } & \text { para } \\
\text { melhorar } & \text { a } \\
\text { adaptação } & \text { ao } \\
\text { tratamento } & \text { com } \\
\text { CPAP } & \text { em } \\
\text { pacientes } & \text { com } \\
\text { SAHOS. } & \end{array}$ & $\begin{array}{l}\text { Estabelecer } \\
\text { objetivamente o grau de } \\
\text { uso do CPAP, uma vez } \\
\text { que foi demonstrado que } \\
\text { os pacientes } \\
\text { superestimam a média } \\
\text { de horas de uso em mais } \\
\text { de uma hora por noite. }\end{array}$ \\
\hline & $\begin{array}{l}\text { Gisele S. Moura L. } \\
\text { Neves, Philippe }\end{array}$ & $\begin{array}{l}\text { Transtornos do } \\
\text { sono: atualização } \\
(1 / 2)\end{array}$ & $\begin{array}{l}\text { Atualizar outra revisão } \\
\text { publicada por este grupo } \\
\text { de pesquisadores sobre }\end{array}$ \\
\hline
\end{tabular}




\begin{tabular}{|c|c|c|c|}
\hline 2017 & $\begin{array}{l}\text { M., Marleide da } \\
\text { Mota G. }\end{array}$ & & $\begin{array}{l}\text { sono e epilepsia, sendo a } \\
\text { principal preocupação } \\
\text { desta apresentação e } \\
\text { discussão da nova } \\
\text { classificação da terceira } \\
\text { edição da classificação } \\
\text { internacional de } \\
\text { transtornos do sono. }\end{array}$ \\
\hline 2013 & $\begin{array}{l}\text { Gisele S. Moura L. } \\
\text { Neves, Andre S. } \\
\text { G., } \quad \text { Patricia } \\
\text { Florido3, M. da } \\
\text { Mota G. }\end{array}$ & $\begin{array}{l}\text { Transtornos do } \\
\text { sono: visão geral }\end{array}$ & $\begin{array}{l}\text { compreender as } \\
\text { características do ciclo } \\
\text { vigília-sono e das fases } \\
\text { do sono, além de suas } \\
\text { manifestações, } \\
\text { conhecer a classificação, } \\
\text { as abordagens } \\
\text { diagnósticas e os } \\
\text { tratamentos } \\
\text { (farmacológicos ou não) } \\
\text { dos TS, para melhor } \\
\text { controlá-los, } \\
\text { principalmente } \\
\text { relação a adultos }\end{array}$ \\
\hline
\end{tabular}

Fonte: Elaborado pelos autores (2019)

Ao analisar os estudos, a fim de responder o problema de pesquisa: Quais os benefícios que o CPAP traz para os portadores da SAOS que usam o dispositivo? emergiram as categorias que serão apresentadas no Quadro 2.

Quadro 2: Distribuição das categorias temáticas segundo as amostras dos estudos. São Paulo - SP, Brasil, 2019.

\section{Benefícios do tratamento com uso contínuo do CPAP}




\section{Perfil e características dos portadores da SAOS}

\section{Possíveis complicações nos pacientes portadores de SAOS}

Benefícios da prática do exercício físico como aliado na melhora da qualidade de vida (QV)

Síndrome da Apneia Obstrutiva do sono e ronco

Fonte: Elaborado pelos autores (2019).

\subsection{BENEFÍCIOS DO TRATAMENTO COM USO CONTÍNUO DO CPAP}

Estudos descrevem que o CPAP é a primeira opção de tratamento para os portadores da SAOS. Se o paciente aderir ao tratamento de forma correta, ocorrerá melhora do controle glicêmico, diminuição nos episódios de roncos, melhora da qualidade do sono e a prevenção de possíveis riscos cardiovasculares (LACERDA et al, 2013). Autores relatam que alguns pacientes não aderem ao dispositivo pelo não conhecimento completo de sua enfermidade, falta de programas educativos que orientem o uso correto do CPAP e devido à condição socioeconômica. Esses fatores comprometem a complacência ao tratamento (ANDRADE; PEDROSA, 2016; ALVES; RAMOS; VOLPATO, 2016).

\subsection{PERFIL E CARACTERÍSTICAS DOS PORTADORES DA SAOS}

Como resultado da pesquisa bibliográfica, procurou-se enfatizar as alterações mais significativas nos portadores da SAOS. Pesquisas mostram que os portadores da síndrome são acometidos em grande maioria entre os 40 e 60 anos. Na maior parte dos casos, os pacientes têm características alteradas, tais como: alterações craniofaciais, obesidade, hipertensão e em casos de circunferência do pescoço superior a $40 \mathrm{~cm}$ deve-se levar a suspeita de possíveis desordens respiratórias relacionadas ao sono (MOURA et al, 2017). 


\subsection{POSSÍVEIS COMPLICAÇÕES NOS PACIENTES PORTADORES DE SAOS}

Estudos evidenciam que a SAOS é uma condição crônica complexa que pode causar problemas físicos, psicológicos e sociais aos seus portadores. Apesar da maioria dos sintomas ser de origem respiratória, pesquisas têm consistentemente mostrado que os portadores da SAOS têm alto risco para o desenvolvimento de doenças cardiovasculares. Dentre elas destaca-se a hipertensão arterial, insuficiência cardíaca, doença arterial coronariana e arritmias, além de evidenciar que essas doenças são a maior causa atual de morbimortalidade no mundo (PACHECO; ANJOS; MAIA, 2015; CAMPOSTRINI; PRADO; PRADO, 2014). Outros autores apontam os riscos de acidentes automobilísticos e ocupacionais, além de afastar o portador do convívio social e piorar consideravelmente a qualidade de vida (MOURA et al, 2017).

\subsection{BENEFÍCIOS DA PRÁTICA DO EXERCÍCIO FÍSICO COMO ALIADO NA MELHORA DA QUALIDADE DE VIDA (QV)}

Os achados apresentados descrevem que a prática de atividade física promove a redução de peso, fortalecendo o tônus da musculatura dilatadora das vias aéreas superiores, o que melhora a eficiência do consumo de $\mathrm{O}^{2}$ bem como há a regulação do sono. Outras literaturas relatam que a maioria dos portadores que praticam exercícios físicos obtiveram diminuição dos agravos da doença e aumento significativo da eficiência do sono (NOGUEIRA; BORSINI; NIGRO, 2016).

\subsection{SÍNDROME DA APNEIA OBSTRUTIVA DO SONO E RONCO}

Em todas as literaturas usadas o ronco é citado como um dos sintomas da síndrome, sendo ele um fenômeno biofísico que ocorre durante o sono e nem sempre está relacionado com as apneias, mas é um sinal de alerta. O ronco é uma manifestação sonora de uma deficiência das vias aéreas superiores e quando relacionado a outros sintomas, como a queda das atividades diurnas, deve ser examinado e investigado mais a fundo, pois ele deixou de ser considerado apenas como constrangimento aos 
pacientes, pois pode, também, acarretar em consequências para a saúde do roncador (NEVES; MACEDO; GOMES, 2017; NEVES et al, 2013).

\section{DISCUSSÃO}

Novos estudos analisaram a prática da atividade física como um aliado contra a SAOS. Alguns autores discorrem que a modalidade terapêutica é de baixo custo e eficiente no atraso ou diminuição de danos decorrentes da SAOS. Em uma pesquisa clínica com pacientes adultos foram apresentadas peculiaridades significativas na redução do Índice de Apneia/Hipopneia (IAH), sonolência diurna, aumento da efetividade do sono e do pico de oxigênio. Os pesquisadores identificara , também, que os pacientes que possuem regularidade na prática de exercícios físicos obtiveram uma redução de $32 \%$ de seus sintomas, mas outros autores relatam que a atividade física possui benefícios, porém não altera, de forma significativa, o quadro clínico, pois existem vantagens indiretas que apenas amenizam os sinais e sintomas da patologia atuando, apenas, como auxílio contra a doença (ANDRADE; PEDROSA, 2016).

De acordo com estudiosos, a qualidade do sono está relacionada com o período sonovigília. Porém essa qualidade pode ser afetada por patologias como a SAOS, o que compromete consideravelmente a eficiência do sono. Em nossos achados detectamos que houve piora devido à não aderência do CPAP por deficiência da adequação do dispositivo ao paciente e em razão da falta de treinamento, o que dificulta sua aderência e, consequentemente, a adaptação do portador. Alguns pesquisadores relatam dificuldades como resistência das vias aéreas superiores; menor idade; menor gravidade da doença e que pacientes que vivem sozinhos não sabem manusear o dispositivo. Assim como outros autores descrevem que para uma maior aceitação é preciso o desenvolvimento de programas educativos, maior entendimento sobre sua patologia e compromisso clínico dos portadores e da equipe multiprofissional (NOGUEIRA; BORSINI; NIGRO, 2016).

Diante dos resultados obtidos, é notório que a SAOS é um fator predisponente para o desenvolvimento de complicações cardiovasculares, sendo ainda mais comum quando o paciente tem fatores de risco pré-existentes associados, tais como: idade, 
gênero, alterações craniofaciais, fatores genéticos, tabagismo e ingestão de álcool. Além disso, os autores também evidenciam altos riscos de acidentes automobilísticos e ocupacionais. Essa evidência é associada aos sintomas da SAOS, como a sensação de noite mal dormida, sonolência excessiva diurna, falta de concentração. Esses fatores propiciam riscos de acidentes e diminuem drasticamente a qualidade de vida do portador, afetando, também, a vida social. A SAOS é uma síndrome que apresenta diversos sintomas, muitas vezes ignorados por seu portador, até por falta de conhecimento, dificultando, assim, seu diagnóstico.

O ronco é um dos principais sintomas e deve ser tratado com tal seriedade, sendo divulgado para a população leiga, para que ela se atente aos episódios frequentes de ronco associado a outros sintomas, procurando, assim, a equipe multiprofissional. De acordo com estudos já realizados, encontramos indícios da eficácia do tratamento adequado na diminuição dos sintomas e melhora significativa na qualidade de vida dos portadores. Porém, apesar dos bons resultados já obtidos, ainda existem pontos da SAOS pouco explorados. Como exemplo podemos citar a dificuldade do diagnóstico. Autores relatam que muitos portadores não são diagnosticados pelo fato dos sintomas como ronco e sonolência diurna serem considerados algo comum na rotina corrida das pessoas atualmente.

A descoberta precoce da doença e adesão prévia ao tratamento impede muitas complicações na saúde e na vida dos pacientes. Então, como sugestão para futuros estudos, indicamos a necessidade da criação de novos questionários e protocolos e que estes sejam aplicados desde a atenção primária de forma criteriosa. É uma estratégia para que a população esteja mais aberta em relação a essa patologia, e, assim, procure pelos profissionais responsáveis. São necessários, também, novos métodos de avaliação inseridos na equipe multiprofissional para que haja um diagnóstico mais rápido e preciso assim como para que o tratamento seja iniciado mais rapidamente. Isso, consequentemente, acarretará a melhora da qualidade de vida dos portadores. 


\section{CONSIDERAÇÕES FINAIS}

Em vista dos argumentos apresentados, gostaríamos de destacar a relevância do assunto abordado nesta pesquisa, pois é um tema pouco explorado e de alta prevalência na sociedade, provavelmente os números levantados em pesquisas são errôneos e menores do que a realidade. A SAOS é uma doença de difícil diagnóstico e muitos portadores não têm e provavelmente nunca chegarão a um diagnóstico. Os pacientes acometidos pela SAOS, em sua grande maioria, são pessoas entre 40 e 60 anos e muitos têm características físicas alteradas. Dentre elas podemos citar as alterações craniofaciais, obesidade e hipertensão, além de apresentarem sintomas como: ronco, sonolência diurna excessiva, sensação de sono não reparador, episódios de apneia e padrão respiratório anormal.

Essa dificuldade no diagnóstico se deve aos sintomas apresentados por seus portadores serem ignorados e julgados como rotineiros bem como devido ao fato de que o método diagnóstico, a polissonografia, considerada mundialmente "padrãoouro", trata-se de um exame de alto custo e difícil acesso nas redes públicas de saúde. Após o diagnóstico, a melhor forma de tratamento é o CPAP. Trata-se de um aparelho de pressão positiva e contínua nas vias respiratórias que atua por meio de uma máscara. O que determina a quantidade de ar necessária para cada paciente é a polissonografia. Com relação aos resultados obtidos, podemos destacar a importância de treinamento e especialização para profissionais da saúde sobre o tema, pois esse distúrbio é de extrema importância para saúde pública e ocasiona sérios riscos à vida do paciente.

Quando a SAOS não é diagnosticada e tratada previamente é causadora de muitas complicações para o portador, por isso é importante a ampliação dos serviços de atendimento aos distúrbios do sono. Levando- se em consideração esses aspectos, consideramos que o assunto deve ser mais divulgado a profissionais de saúde e para população em geral para que possam ter conhecimento acerca dos sintomas e riscos dessa patologia para que, em caso de suspeita, procurem auxílio da equipe multiprofissional o mais breve possível para uma adesão prévia ao tratamento. Haverá, consequentemente, diminuição das complicações advindas da SAOS. O tema 
abre oportunidade para a enfermagem trabalhar com distúrbios do sono, já que não existe uma equipe de enfermeiros específica para trabalhar com essas patologias. Há, também, escassez de pesquisas científicas sobre distúrbios do sono na área da Enfermagem.

\section{REFERÊNCIAS}

ALVES, P. R. R; RAMOS, F. A; VOLPATO, T. B. Qualidade de vida em indivíduos com apneia obstrutiva do sono moderada a grave antes e após tratamento com pressão positiva contínua nas vias aéreas. ABCS Health Sciences, v. 41, n. 3, p. 146-149, 2016.

ANDRADE, F. M. D. de.; PEDROSA, R. P. O papel do exercício físico na apneia obstrutiva do sono. Jornal Brasileiro de Pneumologia, v. 42, n. 6, p. 457-464, 2016.

CAMPOSTRINI, D. D. A; PRADO, L. B. F. do; PRADO, G. F. do. Síndrome da apneia obstrutiva do sono e doenças cardiovasculares. Revista Neurociências, v. 22, n. 1, p. 102-112, 2014.

JÚNIOR, R. J. P. P; FILHO, G. L; FRAGER, L. F. Tratamento da apneia obstrutiva do sono. Revista Brasileira de Hipertensão, v. 20, n. 1, p. 23-33, 2013.

LACERDA, V. M. A. et al. Qualidade de vida (QV) e aspectos clínicos de pacientes com síndrome da apneia obstrutiva do sono (SAOS) em tratamento com pressão positiva contínua nas vias aéreas (CPAP). Revista Brasileira de Qualidade de Vida, v. 5, n. 1, p. 22-30, 2013.

LEITE, A. R. et al. Correlação de Risco entre Síndrome da Apneia Obstrutiva do Sono e Insuficiência Cardíaca na Atenção. International Journal of Cardiovascular Sciences, v. 30, n. 5, p. 459-463, 2017.

MOURA, W. L. et al. Prevalência do risco da Síndrome da Apneia Obstrutiva do Sono na população adulta de uma capital brasileira. RFO, v. 22, n. 3, p. 303-308, 2017. 
NASCIMENTO, A. P. do. et al. Qualidade do sono e tolerância ao esforço em portadores de apneia obstrutiva do sono. Revista Brasileira de Medicina do Esporte, v. 20, n. 2, p. 115-118, 2014.

NEVES, G. S. M. L. et al. Transtornos do sono: visão geral. Rev Bras Neurol, v. 49, n. 2, p. 57-71, 2013.

NEVES, G. S M. L; GOMES, M. D. M. Transtornos do sono: atualização (1/2). Revista Brasileira Neurologia, v. 53, n. 3, p. 19-30, 2017.

NOGUEIRA, J. F.; BORSINI, E; NIGRO, C. Estrategias para mejorar la adaptación al tratamiento con CPAP en pacientes con SAHOS. Revista Americana de Medicina Respiratória, v. 16, n. 4, p. 365-377, 2016.

PACHECO, F. Y. R; ANJOS, E. S. dos.; MAIA, A. B. da. F. Síndrome da apnéia/hipopnéia obstrutiva do sono: artigo de revisão bibliográfica. UNILUS Ensino e Pesquisa, v. 12, n. 29, p. 45-52, 2016.

PEREIRA, A. R. de. J. Síndrome da Apneia Obstrutiva do Sono e Risco Cardiovascular. 2015. 50 f. Dissertação (Mestrado em Medicina) - Universidade de Coimbra. Lisboa, 2015.

SILVA, A. D. L. da. et al. Multidisciplinaridade na apneia do sono: uma revisão de literatura. Revista CEFAC, v. 16, n. 5, p. 1621-1626, 2014.

TASSINARI, C. C. et al. Capacidade funcional e qualidade de vida entre sujeitos saudáveis e pacientes com apneia obstrutiva do sono. Medicina (Rib Preto), v. 49, n. 2, p. 152-159, 2016.

Enviado: Agosto, 2019.

Aprovado: Janeiro, 2020. 